\title{
An algorithm for determining collision probabilities between small solar system bodies
}

\author{
S.P. Manley ${ }^{1}$, F. Migliorini ${ }^{1,2}$, and M.E. Bailey ${ }^{1}$ \\ 1 Armagh Observatory, College Hill, BT61 9DG, Northern Ireland, UK \\ e-mail: spm@star.arm.ac.uk \\ 2 Osservatorio Astronomico di Torino, Strada Osservatorio 20, I-10025 Pino Torinese (TO), Italy
}

Received March 3; accepted June 26, 1998

\begin{abstract}
The introduction of surveys (e.g. Spacewatch, OCA-DLR) dedicated to the discovery of asteroids and other small bodies is likely to increase the number of known objects to many times the current figure of roughly 30 000. Previous methods for determining collision probabilities amongst these objects (e.g. those due to Öpik, Wetherill, Greenberg and Kessler) all have idiosyncrasies which make them inappropriate for analyses of interactions between large numbers of solar system bodies. Here we present an adaptation of the Wetherill and Greenberg methods, which avoids approximations made by Öpik but which remains accurate and fast enough in its implementation to allow the direct analysis of the collision probabilities and impact velocities of thousands of potentially colliding objects.
\end{abstract}

Key words: minor planets, asteroids — solar system: general - methods: analytical, numerical

\section{Introduction}

The problem of working out collision rates between solar-system bodies has many applications in theories concerning the long-term physical evolution of objects which make up the solar system. Collision rates are of particular importance in the main asteroid belt (Farinella \& Davis 1992), where it has long been recognized that collisions play a key role; also in the EdgeworthKuiper belt (Farinella \& Davis 1996), in meteoritic astronomy, and in all studies concerning the cratering history of the terrestrial planets. Collision rates are also a key factor in understanding the evolution of the zodiacal cloud and in modelling the development of asteroidal surface regoliths.

Send offprint requests to: S.P. Manley
Algorithms for assessing collision probabilities are also demanded in fields such as NEO studies, and where there are suggestions that previous values of collision probabilities in the main belt (Farinella \& Davis 1992; Vedder 1996, 1998), based on the observed population of bodies larger than $50 \mathrm{~km}$, may not be representative of the collision probability for smaller bodies (Cellino et al. 1996). Resolving the latter question requires a new computational effort based on a much larger sample extending to include the small bodies.

One of the first to provide an analytic expression for the collisional probability between a pair of orbits was Öpik (1951). His approach has serious limitations (e.g. one orbit must be circular), but it has been widely used in the past and is still ideal as a quick estimator of collision rates against the planets, whose orbits are roughly circular. Unfortunately Öpik's method, although elegant and purely analytical, has singularities at the apsides (i.e. when the perihelia or aphelia just touch the circular orbit) and whenever the orbits are coplanar.

An improved technique by Wetherill (1967) allows both orbits to be elliptical, and allows for an assumed constant perihelion precession by assuming that the distribution of the value of the true anomaly of one orbit at the point of intersection with the other is uniform. This assumption allows the probability function to be integrated by Monte Carlo sampling, but for such low-dimensional problems Monte Carlo integrations are relatively inefficient.

Greenberg's (1982) method (cf. Bottke \& Greenberg 1993), which can be viewed as a variation of the ÖpikWetherill schemes, is more comprehensive that either of the above, but the geometry unfortunately makes it difficult to implement. The algorithm which we describe below is based on these methods, but uses different reference geometries in order to reduce programming complexity and improve performance. Following Bottke et al. (1994), our algorithm correctly weights the collision velocities between 
any pair of particles, and hence provides a more accurate determination of the frequency distribution of collision velocities in any particular case.

Another method, implemented by Kessler (1981) and Steel \& Baggeley (1985), evaluates the probability density of the particles around their respective orbits using a simple kinetic theory to determine the collision probability. This method generally produces excellent results, but is comparatively slow. The more basic "particle in a box" methods make no allowance for the specific orbital geometries of individual colliding particles, and while useful for simulating large systems are comparatively inaccurate.

More recent innovations include those by Dell'Oro \& Paolicchi (1997) and Vedder (1996). The former authors introduce the novel concept of estimating the collision probability between a target and a large population of field particles, by selecting only those orbits able to collide with the target, and then adjusting the distribution of such orbits to match the statistical properties of the underlying ensemble. This has the advantage of focusing attention solely on orbits which might collide with a given target, allowing the investigation of different field populations with little additional computational effort. By contrast, Vedder (1996) develops a probabilistic method based on the frequency distribution of close approaches between elliptical orbits. Whereas this provides an interesting alternative to other methods, avoiding singularities associated with some methods and orbital geometries, it is relatively computer intensive and not ideally suited as a quick collision probability estimator.

All these methods assume that the collision probability per unit time is sufficiently small that the interacting particles fully sample the orbital phase space before any collision can take place. However, some pairs of particles may be restricted (e.g. by resonances) to certain regions of phase space, whereas other pairs may undergo significant evolution in elements (e.g. semi-major axis) which are assumed in most methods to be constant or slowly varying. The alternative approach, namely direct numerical integration of the relevant particles, in principle allows a very good determination of the collision probability between two objects, but is very computationally expensive. Such an approach has been used, for example, by Yoshikawa \& Nakamura (1994), and the numerical integration approach has been used in different regimes by other authors (e.g. Michel et al. 1996). There are some problems for which this is the only valid approach, a good example being the case of the Trojan swarms, where all the objects are librating about the Jovian $L_{4}$ and $L_{5}$ Lagrange points (Marzari et al. 1996).

Here we present a numerical-based method for assessing the collision probability between pairs of particles which is applicable to various types of problem. It is fast, depends on simple assumptions, and in general allows an accurate estimation of the collision probability between arbitrary pairs of elliptical orbits.

\section{The method}

\subsection{Assumptions}

The algorithm is designed to work with two arbitrary elliptical orbits. Due to its nature some classes of problem (i.e. objects in secular, mean-motion and Kozai resonances, including extreme cases of $(e, \omega)$-coupling) may be inappropriate for this approach. The basic assumptions involved are:

1. each orbit has fixed semi-major-axis $(a)$, eccentricity (e) and inclination $(i)$;

2 . the rate of variation of the argument of perihelion $(\omega)$ is greater than that of any of the other elements;

3 . the variations of the respective arguments of perihelia are not correlated.

In addition, we assume that the objects are of small size compared to their orbits, and that there is no significant gravitational focusing. The algorithm is readily extended to incorporate the latter, as we show in Sect. 3.

\subsection{Algorithm}

Let us suppose that each orbit has semi-major axis, eccentricity and inclination $\left(a_{1}, e_{1}, i_{1}\right)$ and $\left(a_{2}, e_{2}, i_{2}\right)$ respectively. The longitudes of the ascending nodes $\left(\Omega_{1}, \Omega_{2}\right.$ respectively) are assumed to be fixed, as are the inclinations, and hence so too is the mutual inclination $\Delta i$ of the two orbital planes. We allow the arguments of perihelion of each object, $\omega_{1}$ and $\omega_{2}$, to take all possible values in the range $(-\pi, \pi)$. This defines a two-dimensional phase space in which the differential collision probability at each point, $P=P\left(\omega_{1}, \omega_{2}\right)$, can be evaluated. The total collision probability is then

$P_{\text {tot }}=\int_{-\pi}^{\pi} \int_{-\pi}^{\pi} P\left(\omega_{1}, \omega_{2}\right) \mathrm{d} \omega_{1} \mathrm{~d} \omega_{2}$.

Over most of this phase space the collision probability $P\left(\omega_{1}, \omega_{2}\right)$ is zero because interactions can only occur when the mutual nodes are sufficiently close. Without loss of generality the coordinate system can be chosen so that the origin lies at the focus of the two orbits, the $(x, y)$-plane coincides with the orbital plane of the first orbit, and the $x$-axis lies along the line of intersection of the two orbital planes (Fig. 1). In each case $\omega_{1}$ and $\omega_{2}$ are measured in the orbital plane from the mutual node.

In general, the heliocentric distances $r$ of the ascending or descending nodes are given by

$r=\frac{a\left(1-e^{2}\right)}{1 \pm e \cos \omega}$

and collisions may occur at the ascending node when

$\frac{a_{1}\left(1-e_{1}^{2}\right)}{1+e_{1} \cos \omega_{1}}=\frac{a_{2}\left(1-e_{2}^{2}\right)}{1+e_{2} \cos \omega_{2}}$ 


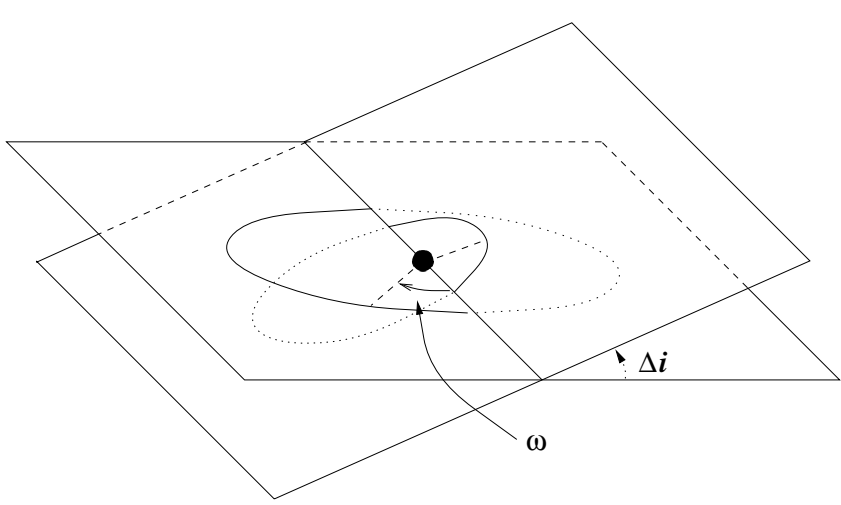

Fig. 1. Intersection of orbital planes, illustrating the mutual inclination, $\Delta i$, and the definition of the argument of perihelion, $\omega$, of one orbit measured with respect to the mutual line of nodes

with a similar expression

$\frac{a_{1}\left(1-e_{1}^{2}\right)}{1-e_{1} \cos \omega_{1}}=\frac{a_{2}\left(1-e_{2}^{2}\right)}{1-e_{2} \cos \omega_{2}}$

for collisions at the descending node. Choosing the ascending node and replacing $a\left(1-e^{2}\right)$ with $p$, and solving for $\cos \omega_{1}$ we obtain:

$\cos \omega_{1}=\frac{p_{1}-p_{2}}{p_{2} e_{1}}+\frac{p_{1} e_{2}}{p_{2} e_{1}} \cos \omega_{2}$.

This relation takes the general form

$\cos \omega_{1}=A+B \cos \omega_{2}$

where

$A=\frac{p_{1}-p_{2}}{p_{2} e_{1}}$

and

$B=\frac{p_{1} e_{2}}{p_{2} e_{1}}$.

This can be differentiated to give

$\frac{\mathrm{d} \omega_{1}}{\mathrm{~d} \omega_{2}}=\frac{B \sin \omega_{2}}{\sqrt{1-\left(A+B \cos \omega_{2}\right)^{2}}}$.

Relations (6) through (8) and corresponding equations for the descending node define the locus of the points in $\left(\omega_{1}, \omega_{2}\right)$-space where collisions may occur, illustrated in Figure 2. Equation (9) is used to determine the tangent and normal to this curve. We note that the curve is symmetrical on both axes, so that looking at one point of intersection there are at most three others with conjugate configurations.

We emphasize that, due to the finite size of real objects, collisions may occur not only on this curve but also in its immediate vicinity, over a range $\Delta \omega\left(\omega_{1}, \omega_{2}\right)$ measured in the $\left(\omega_{1}, \omega_{2}\right)$-plane perpendicular to the curve of intersection. For spherical particles $\Delta \omega$ is proportional to the combined radius of interaction $R=R_{1}+R_{2}$ of the two bodies. Moreover, moving perpendicular to the curve of intersection, it is straightforward to show that the differential collision probability at a distance

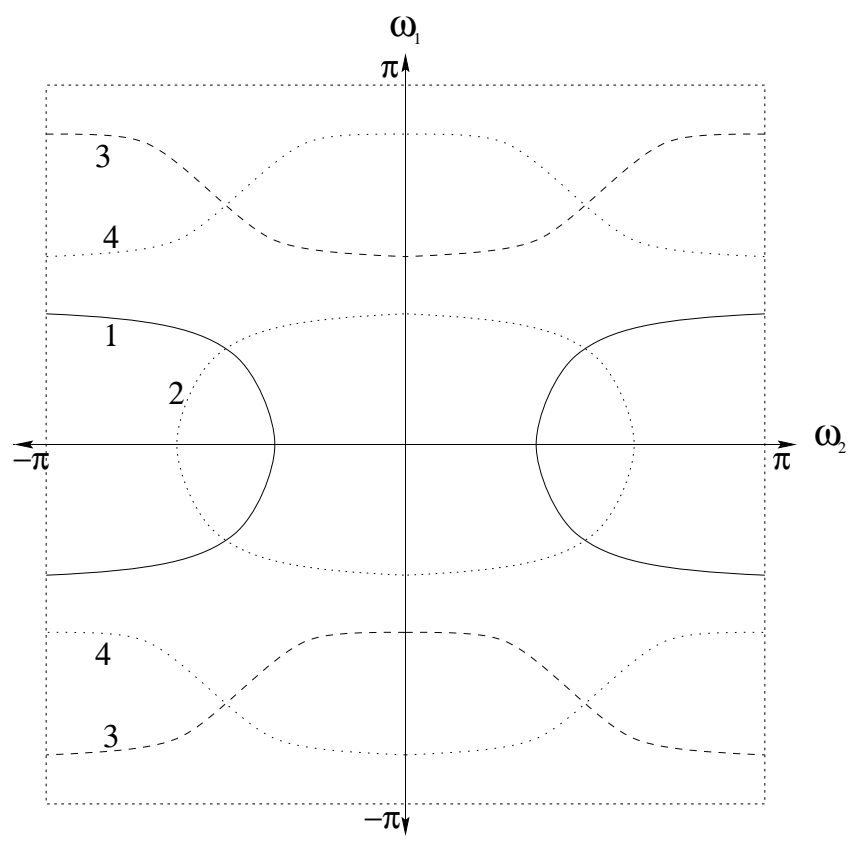

Fig. 2. The figure illustrates the symmetry of the solutions of Eq. (6) in $\left(\omega_{1}, \omega_{2}\right)$-space for two typical pairs of orbits. Each pair of orbits has two solutions. The first pair has solutions denoted " 1 " and " 2 "; the second has solutions denoted " 3 " and " 4 ". In each case these correspond to an intersection at the ascending or descending node. The important points to note are that (i) each solution shows four-fold symmetry, and one can be obtained from the other; and (ii) $\omega_{1}$ is always a monotonically increasing or decreasing function of $\omega_{2}$ in the principal quadrant, depending on whether the intersection occurs at the the ascending or descending node respectively

$|\delta|<\Delta \omega\left(\omega_{1}, \omega_{2}\right)$ from the collision point $\left(\omega_{1}, \omega_{2}\right)$ is $P\left(\omega_{1}, \omega_{2}\right) \sqrt{1-(\delta / \Delta \omega)^{2}}$, so the differential collision probability integrated normal to the curve of intersection is $\frac{\pi}{2} P\left(\omega_{1}, \omega_{2}\right) \Delta \omega\left(\omega_{1}, \omega_{2}\right)$.

The total collision probability is then calculated by integrating along the curves of intersection, choosing small line elements of each curve, determining their dimensions and summing their resulting contributions to the total probability. The direction along each curve of intersection is chosen using $d \omega_{1} / d \omega_{2}$ from Eq. (9). The stepping can be mirrored to allow for the symmetry, but differing geometry (associated with the particles' relative velocities at collision) means that $P$ and $\Delta \omega$ may be different for different mirrored points.

Determination of $P\left(\omega_{1}, \omega_{2}\right)$ and $\Delta \omega\left(\omega_{1}, \omega_{2}\right)$ requires a model of the encounter geometry. We adopt a simple vector approach in which each body is assumed to move at a constant velocity, denoted $\boldsymbol{v}_{1}$ and $\boldsymbol{v}_{2}$ respectively, during the close encounter. The respective vectors (see Fig. 3 ) are determined using the classical formulae for heliocentric elliptical motion (Roy 1988):

$v^{2}=\mu\left(\frac{2}{r}-\frac{1}{a}\right)$ 


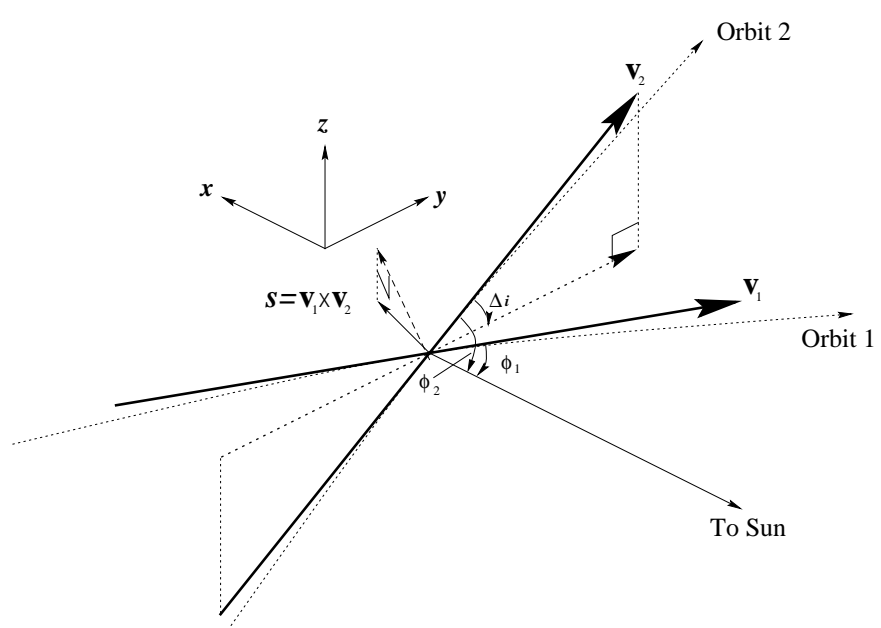

Fig. 3. Diagram to illustrate the simplified vector encounter geometry described in the text

where $\mu=G(M+m)$ and $G$ is the gravitational constant, $M$ is the mass of the central body and $m$ is the mass of the orbiting particle. If we choose $G=4 \pi^{2}, M=1$ and $m=0$ we have:

$v=2 \pi \sqrt{\left(\frac{2}{r}-\frac{1}{a}\right)}$

and for the angle $\phi$ between the velocity and radius vectors at the node

$\sin \phi=\left(\frac{1+e \cos \omega}{\sqrt{1+e^{2}+2 e \cos \omega}}\right)$.

(Note that $\omega$ is measured from the mutual node, and that for simplicity we have dropped subscripts 1 and 2 from Eqs. (10)-(12).) For the first orbit, which lies in the $(x, y)$ plane, the velocity vector is (Fig. 3 ):

$\boldsymbol{v}_{1}=v_{1}\left(\cos \phi_{1}, \sin \phi_{1}, 0\right)$

and for the second, whose plane is inclined at an angle $\Delta i$ to the first, we have

$\boldsymbol{v}_{2}=v_{2}\left(\cos \phi_{2}, \cos \Delta i \sin \phi_{2}, \sin \Delta i \sin \phi_{2}\right)$.

In order for a collision to take place, both particles must arrive at the point of intersection of their respective orbits at the same moment, within a small time interval $\Delta t$. We use $\Delta t$ to calculate the collision probability $P\left(\omega_{1}, \omega_{2}\right)$ at the point $\left(\omega_{1}, \omega_{2}\right)$ on the curve of intersection, and then use the vectors $\boldsymbol{v}_{1}$ and $\boldsymbol{v}_{2}$ to evaluate the range $\Delta \omega$ associated with this point on the curve.

Moving the origin of the coordinate system to the point at which the two paths cross, the motion of the two particles near the mutual node or collision point can be described by

$b=v_{1} \tau \sin \theta$

where $\theta$ is the angle between the two position vectors $\boldsymbol{r}^{\prime}{ }_{1}$ and $\boldsymbol{r}_{2}^{\prime}$ in the new frame. By replacing the maximum value of impact parameter $b$ which produces a collision, namely
$b=R$ where $R$ is the radius of interaction for both particles (i.e. $R=R_{1}+R_{2}$ for spheres of radius $R_{1}$ and $R_{2}$ respectively), we calculate the maximum value of $\tau$, and hence obtain $\Delta t$ using

$\Delta t=R / v_{1} \sin \theta$.

Since the temporal window for a collision is $2 \Delta t$ per revolution, the differential collision probability per unit time for such intersecting orbits is $P\left(\omega_{1}, \omega_{2}\right)=$ $2 \Delta t /\left(P_{\text {orb }, 1} P_{\text {orb }, 2}\right)$, where $P_{\text {orb }, 1}$ and $P_{\text {orb }, 2}$ denote the orbital periods of each particle.

We now determine the magnitude of the range $\Delta \omega$ perpendicular to the curve of intersection. We define $s$ to be the vector that describes the relative motion of the two encounter vectors as $\omega_{1}$ and $\omega_{2}$ are varied normal to the curve of intersection, and introduce a separation parameter $S$, analogous to the impact parameter $b$, whose value equals $R$ at the extremum $\Delta \omega$. We thus have

$S=\frac{\boldsymbol{s} \cdot\left(\boldsymbol{v}_{1} \times \boldsymbol{v}_{2}\right)}{\left|\boldsymbol{v}_{1}\right|\left|\boldsymbol{v}_{2}\right|}$

where

$\boldsymbol{s}=r\left(0, \delta \omega_{1}+\delta \omega_{2} \cos \Delta i, \delta \omega_{2} \sin \Delta i\right)$

and $\delta \omega_{1}$ and $\delta \omega_{2}$ denote the respective variations of $\omega_{1}$ and $\omega_{2}$ perpendicular to the curve of intersection, with $|\boldsymbol{\delta} \omega|<\Delta \omega$. Here, $r$ is the distance from the Sun where the interaction occurs.

Therefore $\Delta \omega$ is simply calculated from

$\Delta \omega=\frac{R}{S}$

and the total collision probability is evaluated by summing the values of $\frac{\pi}{2} P\left(\omega_{1}, \omega_{2}\right) \Delta \omega\left(\omega_{1}, \omega_{2}\right)$ along the curve of intersection in the $\left(\omega_{1}, \omega_{2}\right)$-plane for a statistically sufficient number of points.

\section{Limitations and problems}

\subsection{Variation in $\Omega$}

The approximations employed in this algorithm are valid for accurate work in many applications, but there are certain classes of orbit for which the approximations break down and may lead to inaccurate results.

First, and perhaps most importantly, the algorithm assumes a fixed inclination between the two orbital planes. In reality, the angle $\Omega$ changes with time and this results in a change of the mutual inclination $\Delta i$.

In order to deal with this we repeatedly use the fixed plane algorithm and integrate across the distribution of $\Delta i$ produced by the variations of $\Omega_{1}$ and $\Omega_{2}$. The integration is carried out over the variable $\Delta \Omega$, the difference between $\Omega_{1}$ and $\Omega_{2}$, using standard one-dimensional methods over the interval $\Delta \Omega=(0,2 \pi)$.

It is easy to show that $\Delta i$ is a simple function of $\Delta \Omega$ such that

$\cos \Delta i=\cos i_{1} \cos i_{2}+\sin i_{1} \sin i_{2} \cos \Delta \Omega$ 
assuming that $i_{1}>i_{2}$.

Taking this into account, it becomes possible to cover the vast majority of orbits, but there remain small regions of configuration space where the algorithm breaks down due to geometric singularities. These singularities do not exist in the real system and arise only as a result of the simplified encounter geometry, namely the approximation of rectilinear relative motion during the close encounter.

\subsection{Variation in other elements}

The algorithm assumes that the elements $a, e$ and $i$ are so slowly varying that they can be assumed to be constant over timescales of interest. In some instances this may not be the case, particularly if the objects are affected by secular perturbations that significantly affect (for example) the eccentricity and inclination, even in cases when the semi-major axis is approximately constant. The point has been discussed by Davis et al. (1992), and in principle such variations could be accommodated within the present algorithm by adding further layers of integration to the model. However, this removes the virtues of simplicity and speed from the model, and would require a more complete dynamical representation of the system. If exact results are required in particular cases, then it may be necessary to return to a computer-intensive numerical integration scheme.

\subsection{Singularities}

It is likely that in any application involving a large number of test bodies some pairs of orbits will lie sufficiently close to singular regions to produce unrealistic results. Singularities are also encountered with other methods, since they are implicit in the assumptions on which the algorithm is based.

Inaccurate results will be produced by pairs of orbits with low relative inclinations, and indeed Öpik suggested that his theory was inadequate for inclinations $<1^{\circ}$. The value of $\Delta \omega$ approaches infinity, at least as evaluated by the simple equations, since as $\Delta i$ approaches zero it becomes impossible to separate the orbits by rotating them around the origin. Examination of the conditions makes it clear that the maximum value of $\Delta \omega$ should be smaller than the size of the phase space over which we are integrating.

The simplest way to prevent this is to ensure that the relative inclination used during the integration is never less than some critical value. The adjustment of the relative inclination should be performed on the inclinations of the input orbits, rather than by limiting $\Delta i$, shifting the values of $i_{1}$ and $i_{2}$ such that Eq. (20) will give a value higher than our enforced limit for all values of $\Delta \Omega$.

Similarly, problems occur when the perihelia or aphelia of either orbit coincide, since for such orbits the separation parameter $S \rightarrow 0$ at this point and therefore $\Delta \omega$ diverges. This is a direct result of approximating the curved trajectories in space at the close encounter by straight lines. For orbits with configurations sufficiently close to this singularity condition the results will be inaccurate.

In the version of the code implemented by the authors these singularities are dealt with by interpolating across the region where the singularity is known to exist. Orbits with $q$ or $Q$ sufficiently close to the $Q$ or $q$ of the other have probabilities computed by taking variations of each orbit with slightly differing elements, the magnitude of these variations being chosen to be comparable to the shortterm variations in the orbital elements introduced by ordinary planetary perturbations. In practical applications we have found that such an approach produces reasonable results.

We emphasize that these singularities, arising from nearly coplanar or tangent orbits, are unphysical, in the sense (as proved by Wetherill 1967) that the corresponding collision integrals are always finite and can in principle be evaluated using other methods. One way of dealing with them is to use a more appropriate numerical integration technique, as was implemented by Farinella \& Davis (1992); another, involving a suitable change of coordinates to remove the improper integral, has been discussed by Milani et al. (1990). In either case, owing to orbital evolution, the singularities are only short-lived, and the method of handling them has to be considered in relation to the physics of the dynamical system.

\subsection{Uniformity of sampling within $\left(\omega_{1}, \omega_{2}\right)$-space}

The present algorithm assumes that the orbits sample $\left(\omega_{1}, \omega_{2}\right)$-space uniformly. It is difficult to provide simple rules to determine whether a pair of orbits satisfies this criterion adequately. For example, both orbits may be in resonances, leading the orbit pairs to have strongly correlated elements. Such orbits may only explore certain regions of phase space, along specific paths, and we can no longer logically assume that every point in $\left(\omega_{1}, \omega_{2}\right)$-space is equally likely. Obviously, for every pair of orbits the evolution of $\omega_{1}$ and $\omega_{2}$ can in principle be determined, but good pairs will explore most of $\left(\omega_{1}, \omega_{2}\right)$-space uniformly. The approximation to this behaviour is that every point in $\left(\omega_{1}, \omega_{2}\right)$-space is equally weighted, as too are the points on the curve of intersection. Unfortunately, the most straightforward way to avoid these difficulties is through a direct numerical integration of the problem orbits, which of course is computationally expensive and is what this and similar methods have been designed to avoid.

\subsection{Gravitational focusing}

The algorithm assumes fixed target radii, but if the bodies have sufficient mass then the effective target area will 
Table 1. Comparison between the total collision probabilities per unit time between various bodies, determined by previous authors and this work. The values refer to encounters between real objects and the hypothetical test body, Astrid, considered by Arnold (1965), Wetherill (1967), and Greenberg (1982). The orbital elements of Astrid are $(a, e, i)=(2.75,0.2727,0.2760)$, and in evaluating the total collision probability we have assumed a combined interaction radius $R=1 \mathrm{~km}$, corresponding to a geometric cross-section for collision $\sigma=\pi \mathrm{km}^{2}$. We emphasize that this fictitious body has no relation to the real asteroid (1128) Astrid. The tabulated values ignore gravitational focusing, and are given in units of $10^{-18} \mathrm{yr}^{-1}$. The columns denoted

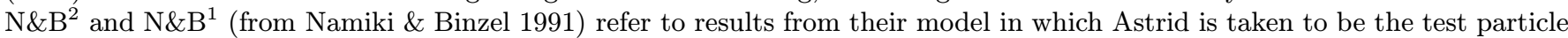
and field particle respectively; the difference between these columns provides an indication of the relative accuracy of their results

\begin{tabular}{|c|c|c|c|c|c|c|c|c|c|c|}
\hline Object & $a$ & $e$ & $i$ & Arnold & Wetherill & 1 Greenberg & $N \& B^{2}$ & $N \& B^{1}$ & $\mathrm{BG}$ & This Work \\
\hline $1948 \mathrm{EA}$ & 2.263 & 0.605 & 0.3226 & 1.92 & 3.10 & 2.49 & 3.03 & 3.19 & 3.20 & 3.20 \\
\hline Apollo & 1.48 & 0.56 & 0.1047 & 3.03 & 4.22 & 3.24 & 3.46 & 3.48 & 3.60 & 3.60 \\
\hline Adonis & 1.97 & 0.78 & 0.0349 & 4.95 & 4.13 & 3.92 & 4.24 & 4.53 & 4.53 & 4.53 \\
\hline $1950 \mathrm{DA}$ & 1.695 & 0.506 & 0.2131 & 2.19 & 3.90 & 3.13 & 3.61 & 3.64 & 3.76 & 3.76 \\
\hline Comet Encke & 2.214 & 0.847 & 0.216 & 2.56 & 3.49 & 2.91 & 3.25 & 3.43 & 3.43 & 3.43 \\
\hline Comet Brorsen & 3.099 & 0.81 & 0.513 & 0.79 & 0.94 & 0.81 & 0.90 & 0.95 & 0.95 & 0.95 \\
\hline Comet Grigg-Mellish & 30.005 & 0.969 & 1.916 & 0.02 & 0.022 & 0.021 & 0.021 & 0.022 & 0.022 & 0.022 \\
\hline Comet Temple-Tuttle & 10.325 & 0.905 & 2.839 & 0.33 & 0.62 & 0.60 & 0.59 & 0.62 & 0.62 & 0.62 \\
\hline Comet Neujmin & 4.931 & 0.588 & 0.066 & 0.83 & 0.89 & 0.74 & 0.92 & 0.90 & 0.94 & 0.94 \\
\hline Comet Schaumasse & 4.059 & 0.705 & 0.209 & 0.9 & 1.16 & 0.91 & 1.09 & 1.15 & 1.15 & 1.15 \\
\hline Comet Pons-Brooks & 17.120 & 0.955 & 1.295 & 0.037 & 0.041 & 0.038 & 0.039 & 0.041 & 0.041 & 0.041 \\
\hline
\end{tabular}

be a function of their relative velocity, owing to their mutual gravitational attraction. For spherical targets with a combined mass $M=M_{1}+M_{2}$ and radius of interaction $R=R_{1}+R_{2}$ the effective cross section, $\sigma_{\text {eff }}$ can be calculated from

$\sigma_{\mathrm{eff}}=\sigma\left(1+\frac{v_{\mathrm{esc}}^{2}}{v_{\mathrm{enc}}^{2}}\right)$

where $v_{\text {enc }}$ is the relative encounter velocity, $\sigma$ is the unfocused cross section (i.e. $\sigma=\pi\left(R_{1}+R_{2}\right)^{2}$ ), and $v_{\text {esc }}^{2}=2 G M / R$. This correction must be applied at each point of the sampled curve, since the relative velocity is a function of $\left(\omega_{1}, \omega_{2}\right)$.

We note that both $P$ (proportional to $\Delta t$ ) and $\Delta \omega$ are proportional to $R$, so the resulting collision probability is proportional to $R^{2}$ and therefore proportional to $\sigma$.

\section{Tests and results}

This algorithm was designed to calculate efficiently the collision probability per unit time between objects moving in arbitrary elliptical orbits. The algorithm has been tested in a variety of ways and against a number of previous results. First we compare our results with those of Wetherill (1967) and subsequent workers, who have determined the intrinsic collision probability between a number of selected comets and asteroids against a hypothetical test body, Astrid', with orbital elements $(a, e, i)=$ (2.75 AU, 0.2727, 0.2760 (rads)). The results are shown in Table 1, which shows excellent agreement with the independent results of Namiki \& Binzel (1991) and Bottke

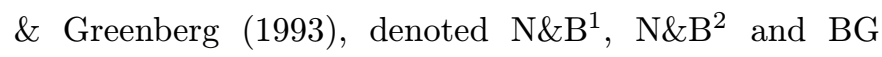
respectively.

Next we evaluated the impact probabilities of selected objects with the Earth. Table 2 shows the comparison between a number of Apollo and Aten asteroids, with orbital elements and other data from Shoemaker et al. (1979) and results from the Öpik method taken from Steel \& Baggaley (1985). Table 3 gives corresponding results for comets, taken from Olsson-Steel (1987), and Table 4 shows the equivalent comparison for long-period comets with extremely high impact probabilities, with elements taken from Marsden \& Steel (1994).

These results are also in good agreement with those of previous authors, with several notable exceptions, namely: (2101) Adonis (Table 2), $1862 \mathrm{II}=\mathrm{C} / 1862 \mathrm{~N} 1$ and $1945 \mathrm{III}$ $=\mathrm{C} / 1945 \mathrm{~L} 1$ (Table 4). The first of these is close to a singularity at low inclination; the second and third have perihelion distances close to that of the Earth. As we have discussed, most methods for evaluating the collision probability experience singularities at very low and very high relative inclinations, and when either the perihelion or aphelion of one orbit is in close proximity to those points in the other orbit. These minor differences show that further work is required in order to determine the precise value of the impact probability for some of these more extreme cases.

We have confirmed that the mean terrestrial impact probability $p_{E}$ for an isotropic flux of nearly parabolic orbits with perihelion distances uniformly distributed in the interval $0-1 \mathrm{AU}$ is $2.1810^{-9}$ per revolution (cf. Weissman 
Table 2. Impact probabilities between representative near-Earth asteroids and the Earth, per billion years, taken from Steel \& Baggaley (1985), compared with results from this paper

\begin{tabular}{lcccrrrrr}
\hline \multicolumn{1}{c}{ Object } & $a$ & Period & $e$ & $i$ & \multicolumn{5}{c}{ Collision probability per billion years } \\
\hline & (AU) & (years) & & (deg) & SB & SWHW & Öpik & This paper \\
\hline (2100) Ra-Shalom & 0.8321 & 0.7590 & 0.4364 & 15.76 & 6.11 & 6.7 & 6.3 & 6.12 \\
(2340) Hathor & 0.8439 & 0.7752 & 0.4498 & 5.86 & 14.71 & 14.0 & 14.0 & 14.71 \\
(2062) Aten & 0.9665 & 0.9502 & 0.1826 & 18.94 & 8.01 & 6.4 & 6.9 & 8.02 \\
(1566) Icarus & 1.0779 & 1.1191 & 0.8268 & 22.91 & 1.77 & 2.0 & 1.6 & 1.77 \\
(1865) Cerberus & 1.0801 & 1.1225 & 0.4669 & 16.09 & 3.14 & 3.1 & 2.5 & 3.14 \\
(2102) Tantalus & 1.2900 & 1.4652 & 0.2984 & 64.02 & 2.50 & 1.5 & 2.5 & 2.49 \\
(1864) Daedalus & 1.4609 & 1.7658 & 0.6148 & 22.16 & 1.27 & 1.6 & 1.0 & 1.27 \\
1937 UB Hermes & 1.6393 & 2.0989 & 0.6236 & 6.22 & 3.50 & 3.7 & 2.2 & 3.49 \\
(1981) Midas & 1.7759 & 2.3666 & 0.6499 & 39.84 & 0.65 & 0.7 & 3.8 & 0.65 \\
(2101) Adonis & 1.8749 & 2.5672 & 0.7638 & 1.36 & 11.53 & 6.3 & 2.9 & 11.53 \\
\hline
\end{tabular}

Table 3. Impact probabilities between representative Earth-crossing comets and the Earth taken from Olsson-Steel (1985) and comparison with this paper. The Olsson-Steel results are given in units per billion orbits and per billion years respectively, and those from this paper are in units per billion years. Note that the orbital elements are those used by Olsson-Steel

\begin{tabular}{lrrrrrrr}
\hline \multicolumn{1}{c}{ Object } & \multicolumn{1}{c}{$a$} & Period & $e$ & $i$ & \multicolumn{2}{c}{ Olsson-Steel results } & This paper \\
\hline & (AU) & (years) & & $(\mathrm{deg})$ & $\left(10^{-9} / \mathrm{rev}\right)$ & $\left(10^{-9} / \mathrm{yr}\right)$ & $\left(10^{-9} / \mathrm{yr}\right)$ \\
\hline 2P/Encke & 2.21 & 3.3 & 0.846 & 11.9 & 3.4 & 1.035 & 1.015 \\
5D/Brorsen & 3.11 & 5.5 & 0.810 & 29.4 & 1.6 & 0.292 & 0.299 \\
3D/Biela & 3.53 & 6.6 & 0.756 & 12.6 & 4.8 & 0.724 & 0.721 \\
8P/Tuttle & 5.73 & 13.7 & 0.823 & 54.5 & 2.9 & 0.211 & 0.279 \\
55P/Tempel-Tuttle & 10.23 & 32.7 & 0.904 & 162.7 & 30.0 & 0.917 & 1.127 \\
12P/Pons-Brooks & 17.20 & 71.3 & 0.955 & 74.2 & 1.5 & 0.021 & 0.021 \\
1P/Halley & 18.00 & 76.4 & 0.967 & 162.2 & 5.0 & 0.065 & 0.065 \\
109P/Swift-Tuttle & 25.00 & 125.0 & 0.960 & 113.6 & 5.4 & 0.043 & 0.049 \\
35P/Herschel-Rigollet & 29.00 & 156.2 & 0.974 & 64.2 & 1.4 & 0.009 & 0.009 \\
\hline
\end{tabular}

1997 and refs. therein), with a mean impact velocity including gravitational focusing of $54.9 \mathrm{~km} \mathrm{~s}^{-1}$. Alternative expressions for the frequency distribution of long-period comets versus perihelion distance (Everhart 1967; Kresák 1978) generally show an increase with perihelion distance. This leads to a higher average terrestrial impact probability, namely: $(2.46,2.36,2.50) 10^{-9}$ per revolution, for what Kresák (1978) respectively calls Everhart's empirical and alternative models, and Kresák's uniform density model. The observed long-period comets, whilst not necessarily representative of the intrinsic near-parabolic flux, show an even stronger increase with perihelion distance towards $1 \mathrm{AU}$, and give a mean impact probability of $3.310^{-9}$ per revolution (cf. Shoemaker 1984; Olsson-Steel 1987).

In order to test the performance of the algorithm with a large ensemble, we examined the set of 682 asteroids with diameters $D>50 \mathrm{~km}$ used by Farinella \& Davis (1992) and later by Bottke et al. (1994) and Vedder (1998). The distribution of collision velocities was determined for every interacting pair and the total summed distribu-
Table 4. Selected long-period comets with high impact probabilities with respect to the Earth and comparison between the results of Marsden \& Steel (1994), denoted MS, and this paper. Note that orbital elements have been taken from Marsden \& Steel. The impact probabilities are quoted in units of $10^{-8}$ per revolution

\begin{tabular}{lccrcc}
\hline Object & $q$ & $e$ & \multicolumn{1}{c}{$i$} & MS & This paper \\
\hline \multicolumn{5}{c}{$(\mathrm{AU})$} & \multicolumn{3}{c}{$(\mathrm{deg})$} \\
\hline 1862 II & 0.9813 & 1.0000 & 172.11 & 26.00 & 7.11 \\
1864 II & 0.9093 & 0.9964 & 178.13 & 10.30 & 10.27 \\
1759 III & 0.9658 & 1.0000 & 175.13 & 6.91 & 6.76 \\
1945 III & 0.9981 & 1.0000 & 156.51 & 2.68 & 1.64 \\
1743 I & 0.8382 & 1.0000 & 2.28 & 2.21 & 2.21 \\
1987 III & 0.8696 & 0.9957 & 172.23 & 2.06 & 2.05 \\
\hline
\end{tabular}

tion is shown in Fig. 4. The mean colision velocity is $5.30 \mathrm{~km} \mathrm{~s}^{-1}$, which may be compared with the values calculated by Farinella \& Davis (1992); Bottke et al. 


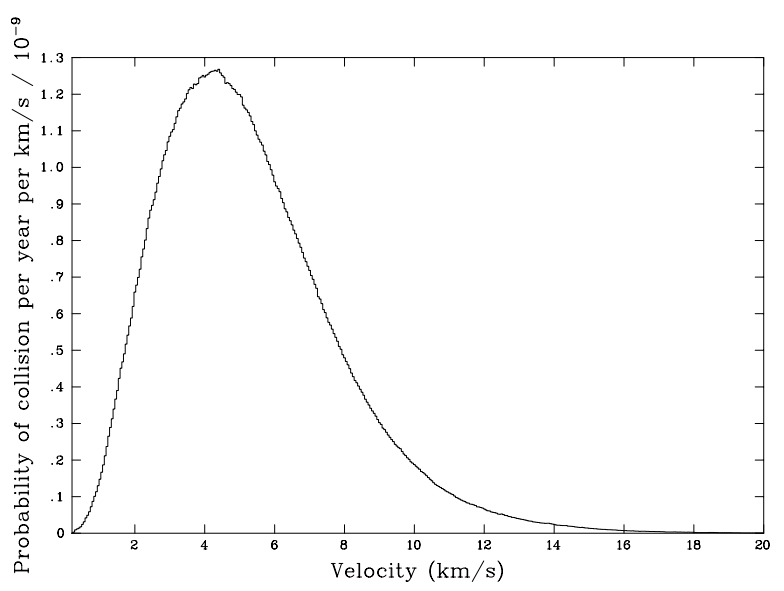

Fig. 4. The figure shows the frequency distribution of collision velocities for main-belt asteroids with diameters $d>50 \mathrm{~km}$

(1994) and Vedder (1998) of $5.81 \mathrm{~km} \mathrm{~s}^{-1}, 5.29 \mathrm{~km} \mathrm{~s}^{-1}$ and $4.22 \mathrm{~km} \mathrm{~s}^{-1}$ respectively. Similarly, the intrinsic collision probability for this sample (assuming a diameter of $1 \mathrm{~km}$ for each object, i.e. corresponding to an interaction crosssection of $\pi \mathrm{km}^{2}$; cf. Table 1 ), is $2.7910^{-18} \mathrm{yr}^{-1} \mathrm{~km}^{-2}$, which may be compared with the values $2.8510^{-18} \mathrm{yr}^{-1}$ $\mathrm{km}^{-2}$ and $3.2710^{-18} \mathrm{yr}^{-1} \mathrm{~km}^{-2}$ reported by Farinella \& Davis (1992) and Vedder (1998) respectively. The method of the latter author appears to lead to a slightly lower mean impact velocity and a slightly higher mean intrinsic collision probability. Use of the present algorithm resulted in a total collision probability for the 682 -asteroid ensemble of $7.2610^{-9} \mathrm{yr}^{-1}$, implying a mean time $\sim 140 \mathrm{Myr}$ for collisions between objects in the ensemble. Collisions between these large objects could result in the formation of asteroid families, and possibly showers of kilometre-sized fragments on Earth-crossing orbits via fast-track resonant dynamical pathways (Zappalà et al. 1998).

\section{Conclusions}

Comparison with other methods shows that the algorithm does not suffer from the same limitations as those of Öpik and Wetherill, and while its assumptions are similar to those of Greenberg the choice of reference frame makes the algorithm faster. Although this algorithm is much slower than use of Öpik's simple equations, it is still fast enough to carry out large numbers of comparisons, typically allowing several comparisons to be made per second on a desktop PC at 4 significant figure accuracy (cf. Vedder 1996, 1998; Dell'Oro \& Paolicchi 1997).

At this precision large-scale surveys of collision rates in the solar system become possible, and the entire population of known asteroids can be analysed in a matter of hours. This opens the door to realistic simulations of collision rates in many new classes of problem, for example detailed particle generation rates resulting from collisions in the zodiacal cloud, asteroid belt and Edgeworth-Kuiper belt. The source code is available on request from the first author.

Acknowledgements. SPM and FM thank the organizing committee of the Maratea NATO Advanced Study Institute "Small Bodies in the Solar System" for support whilst part of this work was carried out; the work was also supported by the EU-HCM contract CHRX-CT94-0445 (FM) and DENI (SPM). The authors thank M. Carpino, J. Fernández and A. Fitzsimmons for helpful comments and discussions, and especially P. Farinella for detailed comments and suggestions which have greatly improved the manuscript.

\section{References}

Arnold J.R., 1965, Astrophys. J. 141, 1536-1547

Bottke W.F., Greenberg, R., 1993, Geophys. Res. Lett. 20, 879-881

Bottke W.F., Nolan M.C., Greenberg R., Kolvoord R.A., 1994, Icarus 107, 255-268

Cellino A., Zappalà V., Migliorini F., 1996, BAAS 28, 10961097

Davis D.R., Farinella P., Carpino M., 1992, Lunar Planet. Sci. Conf. XXIII, 283-284

Dell'Oro A., Paolicchi P., 1997, Planet. Space Sci. 45, 779-784

Everhart E., 1967, AJ 72, 1002-1011

Farinella P., Davis D.R., 1996, Sci 273, 938-941

Farinella P., Davis D.R., 1992, Icarus 97, 111-123

Greenberg R., 1982, AJ 87, 184-195

Kessler D.J., 1981, Icarus 48, 39-48

Kresák L., Pittich E.M., 1978, Bull. Astron. Inst. Czech. 29, 299-309

Marsden B.G., Steel D.I., 1994, In Hazards due to Comets and Asteroids T. Gehrels (ed.). University of Arizona Press, Tucson, U.S.A., p. 221-239

Marzari F., Scholl H., Farinella P., 1996, Icarus 119, 192-201

Michel P., Froeschlé Ch., Farinella P., 1996, A\&A 313, 993-1007

Milani A., Carpino M., Mazari F., 1990, Icarus 88, 292-335

Namiki N., Binzel R.P., 1991, Geophys. Res. Lett. 18, 11551158

Olsson-Steel D.I., 1987, MNRAS 227, 501-524

Öpik E.J., 1951, Proc. Roy. Irish Aca. 54, 165-199

Roy A.E., 1988, Orbital Motion. Adam Hilger

Shoemaker E.M., Williams J.G., Helin E.F., Wolfe R.F., 1979, in Asteroids, Gehrels T. (ed.). University of Arizon Press, Tucson, U.S.A, p. 253

Shoemaker E.M., 1984, in Patterns of Change in Earth Evolution: Dahlem Konjerenzen, Holland H.D., Trendall A.F. (eds.). Springer-Verlag, Berlin, p. 15

Steel D.I., Baggaley W.J., 1985, MNRAS 212, 817-836

Vedder J.D., 1996, Icarus 123, 436-449

Vedder J.D., 1998, Icarus, 131, 283-290

Weissman P.R., 1997, Annal. N.Y. Acad. Sci. 822, 67-95

Wetherill G.W., 1967, J. Geophys. Res. 72, 2429-2444

Yoshikawa M., Nakamura T., 1994, Icarus 108, 298-308

Zappalà V., Cellino A., Gladman B.J., Manley S.P., Migliorini F., 1998, Icarus (in press) 